University of Nebraska - Lincoln

DigitalCommons@University of Nebraska - Lincoln

Nebraska Cooperative Fish \& Wildlife Research Nebraska Cooperative Fish \& Wildlife Research Unit -- Staff Publications

2014

\title{
Habitat structure and body size distributions: cross-ecosystem comparison for taxa with determinate and indeterminate growth
}

\author{
Kirsty L. Nash \\ James Cook University, nashkirsty@gmail.com \\ Craig R. Allen \\ University of Nebraska-Lincoln, callen3@unl.edu \\ Chris Barichievy \\ University of Witwatersrand \\ Magnus Nyström \\ Stockholm University \\ Nicholas A.J. Graham \\ University of Nebraska-Lincoln, nick.graham@lancaster.ac.uk
}

Follow this and additional works at: https://digitalcommons.unl.edu/ncfwrustaff

Part of the Aquaculture and Fisheries Commons, Environmental Indicators and Impact Assessment Commons, Environmental Monitoring Commons, Natural Resource Economics Commons, Natural Resources and Conservation Commons, and the Water Resource Management Commons

Nash, Kirsty L.; Allen, Craig R.; Barichievy, Chris; Nyström, Magnus; and Graham, Nicholas A.J., "Habitat structure and body size distributions: cross-ecosystem comparison for taxa with determinate and indeterminate growth" (2014). Nebraska Cooperative Fish \& Wildlife Research Unit -- Staff Publications. 167.

https://digitalcommons.unl.edu/ncfwrustaff/167

This Article is brought to you for free and open access by the Nebraska Cooperative Fish \& Wildlife Research Unit at DigitalCommons@University of Nebraska - Lincoln. It has been accepted for inclusion in Nebraska Cooperative Fish \& Wildlife Research Unit -- Staff Publications by an authorized administrator of DigitalCommons@University of Nebraska - Lincoln. 


\title{
Habitat structure and body size distributions: cross-ecosystem comparison for taxa with determinate and indeterminate growth
}

\author{
Kirsty L. Nash, Craig R. Allen, Chris Barichievy, Magnus Nyström, Shana Sundstrom \\ and Nicholas A. J. Graham
}

K. L. Nash (nashkirsty@gmail.com)(orcid.org/0000-0003-0976-3197) and N. A. J. Graham, ARC Centre of Excellence for Coral Reef Studies, James Cook Univ., Townsville, QLD, 4811, Australia. - C. R. Allen, US Geological Survey - Nebraska Cooperative Fish and Wildlife Research Unit, Univ. of Nebraska, Lincoln, NE 68583, USA. - C. Barichievy, Ezemvelo KZN Wildlife, Ithala Game Reserve, Louwsberg 3150, South Africa, and: Centre for African Ecology, Univ. of Witwatersrand 2050, Johannesburg, South Africa. - M. Nyström, Stockholm Resilience Centre, Stockholm Univ., SE-106 91, Stockholm, Sweden. - S. Sundstrom, School of Natural Resources, Univ. of Nebraska, Lincoln, NE 68583, USA.

\begin{abstract}
Habitat structure across multiple spatial and temporal scales has been proposed as a key driver of body size distributions for associated communities. Thus, understanding the relationship between habitat and body size is fundamental to developing predictions regarding the influence of habitat change on animal communities. Much of the work assessing the relationship between habitat structure and body size distributions has focused on terrestrial taxa with determinate growth, and has primarily analysed discontinuities (gaps) in the distribution of species mean sizes (species size relationships or SSRs). The suitability of this approach for taxa with indeterminate growth has yet to be determined. We provide a cross-ecosystem comparison of bird (determinate growth) and fish (indeterminate growth) body mass distributions using four independent data sets. We evaluate three size distribution indices: SSRs, species size-density relationships (SSDRs) and individual size-density relationships (ISDRs), and two types of analysis: looking for either discontinuities or abundance patterns and multi-modality in the distributions. To assess the respective suitability of these three indices and two analytical approaches for understanding habitat-size relationships in different ecosystems, we compare their ability to differentiate bird or fish communities found within contrasting habitat conditions. All three indices of body size distribution are useful for examining the relationship between cross-scale patterns of habitat structure and size for species with determinate growth, such as birds. In contrast, for species with indeterminate growth such as fish, the relationship between habitat structure and body size may be masked when using mean summary metrics, and thus individual-level data (ISDRs) are more useful. Furthermore, ISDRs, which have traditionally been used to study aquatic systems, present a potentially useful common currency for comparing body size distributions across terrestrial and aquatic ecosystems.
\end{abstract}

The complexity of community dynamics has driven the search for simple proxies of key life history and ecological traits, measurable across multiple taxa (White et al. 2007). This has led to considerable interest in body size, which correlates with a broad range of species' traits such as home range, dispersal, trophic level, metabolism and extinction risk (Blackburn and Gaston 1994, Woodward et al. 2005). Body size distributions have been used to quantify energy transfer and biogeochemical cycling in ecosystems (Yvon-Durocher and Allen 2012), to examine the division of resources within a community (White et al. 2007), and to quantify the relative resilience of different communities (Peterson et al. 1998).

Habitat and resource availability are thought to be fundamental drivers of body size distributions over ecological timescales (Holling 1992). Consequently, habitat degradation and land use modification will have implications for body size distributions, with knock-on effects for community interactions, ecosystem processes and resilience (Peterson et al. 1998). The discontinuity hypothesis proposes that the interaction between patterns of habitat structure and resources at different scales, and the scale at which species interact with their environment, influences body size distributions within a community (Holling 1992). Such an interaction occurs because resources are patchily distributed so their availability varies among spatial and temporal scales (Wiens 1989), and the scale or spatio-temporal resolution at which an organism perceives its environment and procures resources is a function of its size (Peters 1983). Species are expected to be clustered in aggregations (or modes) along a body size axis corresponding to scales where resources are available, and separated from neighbouring body size aggregations by discontinuities (gaps or troughs), corresponding to scales where resources are limited (Holling 1992).

To date, the discontinuity hypothesis has primarily been tested in terrestrial ecosystems on mammal and avian fauna (Fischer et al. 2008). These studies have predominantly analyzed patterns in the distribution of species' mean 
body masses (hereafter species size relationships (SSRs); Table 1A), and have provided evidence to support the discontinuity hypothesis (reviewed by Nash et al. 2014). Evaluating SSRs demonstrates how patterns of habitat structure influence associated communities via the availability of niches for different sized species (Robson et al. 2005). However, species size relationships do not account for species' abundances. Distributions quantifying the abundance of different sized species provide an alternative index (hereafter termed species size-density relationships (SSDRs); Table 1B; White et al. 2007). This approach allows examination of how resources are distributed among species, or which size classes predominantly drive energy flow within a system (Ernest 2013). This is important as incorporating abundance and examining how resources are apportioned among size classes may provide a more appropriate test of the discontinuity hypothesis (Thibault et al. 2011).

There are two key assumptions to using both SSRs and SSDRs: 1) summarising size information at the species-level is more informative than using individual-level size data for understanding community structure (Doledec and Statzner 1994), and 2) mean body mass is an appropriate metric to represent the size of a species. The first assumption has underpinned much of the terrestrial body size literature, and is appropriate where there are close ties between species identity, and key life history and ecological traits such as size and mobility, meaning that species-level data is representative of individuals within a population (Doledec and Statzner 1994). The second assumption should hold for taxa with determinate growth and where parental care means that predominantly adults are interacting directly with resources available in their environment, giving a narrow range of body sizes from which to calculate the summary metric. Importantly, variation in the mean body masses among species must exceed size variability within species (Robson et al. 2005).

Little research regarding the discontinuity hypothesis has been carried out in aquatic systems or for taxa exhibiting indeterminate growth such as fish (but see Havlicek and Carpenter 2001, Nash et al. 2013), despite considerable evidence that habitat is important in structuring fish communities (Graham and Nash 2013). It is unlikely that the assumptions underlying SSRs and SSDRs will hold for fish. There has been considerable research suggesting that aquatic communities are strongly size structured, and that individual size may be more informative than specieslevel data in understanding the functioning of aquatic ecosystems (Shurin et al. 2006). Furthermore, unlike many terrestrial vertebrates, individual fish may vary over orders of magnitude in length during the course of their life (Webb et al. 2011), undergo significant ontogenetic changes in habitat and resource requirements (Green and Bellwood 2009), and fish often do not exhibit any form of parental care (Smith and Wootton 1995). Thus, size variability within species may exceed variation among species, such that

Table 1. Different indices of body size distribution considered in this study. Key features and their value in examining the distribution of resources within communities are presented.

\begin{tabular}{lll}
\hline Body mass distribution indices & Description & Distribution of resources \\
\hline A. Species size relationship (SSR) & $\begin{array}{l}\text { - Size is aggregated at the species-level } \\
\text { - Species-level presence-absence data }\end{array}$ & $\begin{array}{c}\text { Examines how resources are distributed across } \\
\text { size classes, providing niches and driving the } \\
\text { number of species within different size } \\
\text { classes. }\end{array}$ \\
Log meater relative weight given to species & & \\
&
\end{tabular}

B. Species size-density relationship (SSDR)

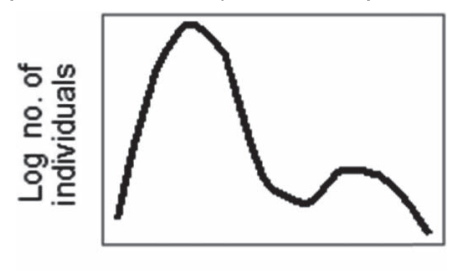

Log mean body size

C. Individual size-density relationship (ISDR)

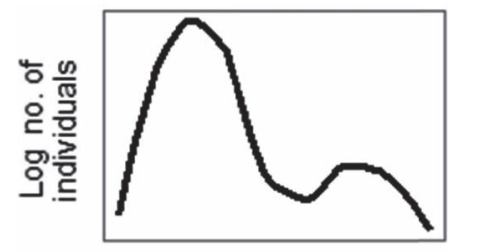

- Size is aggregated at the species-level

- Species-level abundance data

- Greater relative weight given to species identity versus individual size
- Size is presented at the individual-level

- Individual-level abundance data

- Greater relative weight given to individual size versus species identity.
Examines how resources are distributed across size classes, driving abundance of species within different size classes.

Log body size

Examines how resources are distributed across size classes, driving abundance of individuals within each size class. 
species' mean body size may not be an appropriate metric to represent the size of individuals within a population. As a result, there is a need to investigate appropriate indices for use when examining the relationship between habitat and the shape of fish size distributions.

In studies of fish where species identity is of interest, maximum and asymptotic species' body sizes have been suggested as appropriate alternatives to mean size (Jennings et al. 2001). These metrics may be particularly useful in the context of evaluating habitat-body size relationships, as maximum size is likely to be directly influenced by habitat structure in taxa with indeterminate growth (Cumming and Havlicek 2002). However, two issues arise from using maximum length, 1) fish exhibit growth patterns driven by location, latitude and exposure to fishing pressure (Choat and Robertson 2002, DeMartini et al. 2008), so obtaining maximum size data from published sources may introduce bias, and 2) species' maximum size is a summary metric and may not represent intra-specific size variability any better than species' mean size.

For communities where greater relative weight is given to individual body size rather than species-specific traits, a distribution quantifying the abundance of different sized individuals may be a more appropriate body size index (hereafter termed individual size-density relationships (ISDRs); Table 1C; White et al. 2007). This approach examines how resources are divided among individuals within different size classes regardless of an individual's taxonomic affinity. Individual- versus species-level indices have often been applied to different sides of a marine-terrestrial disciplinary divide (size vs species, respectively), and are not generally compared within studies (but see Reuman et al. 2008, O'Gorman and Emmerson 2011). As a result, there has been a lack of clarity regarding the shape of body size distributions and reinforcement of the perspective that marine and terrestrial systems are fundamentally different (White et al. 2007, Webb et al. 2011). This problem has been compounded by a dearth of comparative studies examining body size patterns across multiple ecosystems (but see Petchey and Belgrano 2010, Webb et al. 2011), or among taxa with determinate versus indeterminate growth patterns (but see Forys and Allen 2002).

After selecting the appropriate size distribution index (Table 1), patterns in the size distributions may be analysed in a number of ways (Nash et al. 2014). In the context of the discontinuity hypothesis, studies have primarily looked for discontinuities within size distributions (Fig. 1A; Holling 1992). This approach may be used on either species- or individual-level data, and any abundance information (if present) is ignored; the analysis purely searches for gaps in the distribution. However, an alternative approach is to assess modality patterns in size distributions that incorporate abundance information (Fig. 1B; Xu et al. 2010). These two analytical approaches represent contrasting ways of evaluating body size distributions because they focus on different hypotheses regarding the mechanisms driving the patterns. Multi-modality suggests a concentration of available resources within each mode providing an attractor allowing greater abundances within size classes that utilise resources at coincident scales (Xu et al. 2010). In contrast, discontinuities suggest scales where resources are
(A)

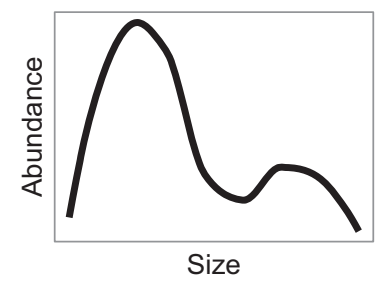

(B)

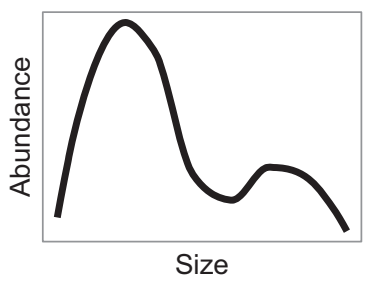

Figure 1. Different analytical approaches for evaluating patterns in body size distributions. (A) Analysis looks for the presence of discontinuities or gaps (red bar) in size distributions. This approach may be used for either distributions that incorporate abundance information (e.g. ISDRs) or those that do not (SSRs) because abundance information is ignored; the analysis solely searches for gaps in the distribution. (B) Analysis evaluates abundance patterns, looking for modes (red bar) and the distribution of abundances across size classes. This approach may only be used for distributions that incorporate abundance information (SSDRs and ISDRs).

absent and thus body size classes that utilize resources at those scales are empty (Holling 1992). The relevance of the two approaches (Fig. 1) in the context of the three size distribution indices (Table 1) has significant implications for understanding what drives community body size distributions, and has not been adequately assessed to date.

The aim of our cross-ecosystem study is to assess if determinate versus indeterminate growth patterns influence the appropriateness of the three different size distribution indices and two distinct analysis methods for detecting habitat effects on body size distributions. Specifically, we examine whether bird or fish communities in habitats of contrasting condition are better differentiated by specieslevel size data analysed for discontinuities, species-level size and abundance data analysed for abundance and modality patterns, individual-level size and abundance data analysed for discontinuities, or individual-level size and abundance data analysed for abundance and modality patterns (Table 2). Our results may be used to develop predictions regarding community, species and individual responses to future environmental change such as habitat degradation and land use modification; specifically the vulnerability of particular size classes and species. Although we focus on habitat as a driver of body size distributions, the approaches may be applicable to research looking at a range of contrasting and complementary drivers such as competitive interactions and biogeography (Allen et al. 2006).

\section{Material and methods}

Two woodland bird (Mount Lofty Ranges and Borneo Upland Forest) and two coral reef fish (Seychelles and the Great Barrier Reef, GBR) datasets were used in the study. Each dataset detailed the bird or fish communities found within multiple habitat types of a particular ecosystem. The Mount Lofty Ranges bird data encompassed two habitat types (stringybark and gum; Possingham et al. 2004). The Borneo Upland Forest bird data covered three habitat 
Table 2. Combinations of the three size distribution indices and two analytical approaches used in the four analyses comparing body size distributions among different habitat types.

\begin{tabular}{|c|c|c|c|}
\hline & & \multicolumn{2}{|c|}{ Analytical approach } \\
\hline & & $\begin{array}{c}\text { Discontinuity } \\
\text { patterns }\end{array}$ & $\begin{array}{c}\text { Abundance } \\
\text { patterns }\end{array}$ \\
\hline \multirow[t]{3}{*}{$\begin{array}{l}\text { Distribution } \\
\text { index }\end{array}$} & $\begin{array}{l}\text { Species size relationship } \\
(\text { SSR })^{\mathrm{a}}\end{array}$ & analysis 1 & b \\
\hline & $\begin{array}{l}\text { Species size-density } \\
\text { relationship (SSDR) }\end{array}$ & c & analysis 2 \\
\hline & $\begin{array}{l}\text { Individuals size-density } \\
\text { relationship (ISDR) }\end{array}$ & analysis 3 & analysis 4 \\
\hline
\end{tabular}

adistributions were based on either mean mass, maximum mass recorded in the literature, or maximum observed mass.

babundance data is not present in species size relationships and thus abundance patterns could not be evaluated.

canalysing discontinuity patterns in species size-density relationships is equivalent to analysing discontinuity patterns in species size relationships (analysis 1), therefore this combination of approaches was redundant.

types (unlogged, logged in 1993, and logged in 1989; Cleary et al. 2007). The Seychelles coral reef fish data incorporated three habitat types (coral dominated, algal dominated, granitic reefs; Nash et al. 2013), and the GBR coral reef fish data encompassed three habitat types (undisturbed, disturbed, recovering; Graham et al. unpubl.). Full details of the datasets, the habitats, and the methods used to collect them are provided in the Supplementary material Appendix 1 Text A1. The various habitat types possessed distinct patterns of cross-scale habitat structure. The body mass distributions of communities from sites within the same habitat type, and thus with similar cross-scale patterns of structure, were expected to be more similar than those from habitats with different structural patterns.

Bird and fish communities were chosen because: 1) they are dominant, species-rich vertebrate groups in their respective ecosystems; 2) they have been the focus of complimentary studies on body mass distributions examining occupancy and abundance patterns (Webb et al. 2011); and 3) our aim was to determine the appropriateness of different approaches for detecting habitat effects on body size distributions rather than to test the discontinuity hypothesis per se; therefore it was important to choose taxa and systems where habitat is known to have a strong influence on body size, and thus the signature of habitat effects should be evident within the size distributions. Most research on the discontinuity hypothesis has been performed on birds, so their relationship to habitat and patterns of discontinuities are well studied. Furthermore, examples from the wider literature have shown that woodland birds are influenced by physical habitat structure (De la Montaña et al. 2006). Similarly, the influence of habitat structure on coral reef fish communities has been particularly well documented (Graham and Nash 2013), and the availability of habitat correlates with fish size (Nash et al. 2013).

\section{Data analysis}

Body mass was used for all size measurements in the analyses. Individual body sizes were not recorded in the bird surveys, and indeed are rarely assessed in bird studies (Ernest 2013) due to the difficulty of estimating the sizes of cryptic species. Therefore, mean body mass data for each bird species were sourced from the Handbook of Avian Body Masses, averaging across estimates where separate male and female records were presented (Dunning Jr. 2008). In addition, maximum recorded body mass of each species were sourced from Dunning Jr. (2008), where available. Thibault et al. (2011) present a method for constructing individual size distributions for bird communities using published mean size and variance data for each species. Information on the variance of some species is not provided by Dunning Jr (2008), therefore species mean body mass data were used to calculate the variance of the mass for each species using the scaling relationship $\operatorname{var}($ mass $)=0.0055 \times$ mean $(\text { mass })^{1.98}$. This relationship is based on the mean-variance relationship of 376 bird species $\left(R^{2}=0.92\right.$; Thibault et al. 2011). Individual body sizes were generated for each dataset by randomly drawing the observed number of individuals from a normal distribution with the estimated mean and variance values of each species. As this method assumes normal distributions and is based on summary statistics it only provides an estimation of the likely size distribution within a community. However, by accounting for intraspecific variability it is more representative of individual size distributions than mean data alone, and this approach has successfully been used to highlight consistency in the shape of bird community ISDRs at macroecological scales (Thibault et al. 2011). For fish, individual length data were recorded in the field, therefore individual, mean and maximum observed body masses were calculated using length: body mass conversions available from FishBase (Froese and Pauly 2012). In addition, maximum recorded body mass of each species were sourced from FishBase (Froese and Pauly 2012).

A critical issue when studying body size distributions is how to effectively compare different distributions. Traditionally, such comparisons have relied on visual assessments (Holling 1992), which are subjective and may not detect key similarities and differences. More recently, comparisons have been made using nested mixture models (Xu et al. 2010) but these rely on a priori decisions regarding the shape of the distributions, or using univariate approaches such as phi correlations (Forys and Allen, 2002) and distribution overlap indices (Ernest 2005). Non-metric multidimensional scaling (nMDS) is a multivariate approach that is commonly used to compare either presence-absence or abundance of species among sites. In this study this approach was extended to allow a comparison of the patterns in the body size distributions among sites. Analysis of similarities (ANOSIM) was used to statistically test for differences in size classes among sites of distinct habitat types (following Hua et al. 2013).

Four groups of analyses were conducted on each dataset, comparing either fish or bird communities among sites of different habitat types, for example comparing the size distributions of bird communities among sites in stringybark and gum habitat (Lofty Ranges dataset). These four groups of analyses evaluated different combinations of the three types of body mass distribution and the two 
analytical approaches (Table 2). ANOSIM significance values will be influenced by the number of replicates within each analysis, therefore Global $\mathrm{R}$ values from the ANOSIM results were used to provide a comparative measure of the strength of the differentiation between habitat types for each analysis (Clarke and Warwick 2001).

\section{Analysis 1 and 3}

For each dataset, discontinuities (Fig. 1A) were evaluated in the species size relationships (analysis 1 ) or the individual size-density relationships (analysis 3) of either the bird or fish community at each site, using the gap rarity index (GRI). The GRI compares the differences between body masses of observed data with those of a null model to assess whether there are significant discontinuities or 'gaps' in the observed size distribution. The null model is produced by fitting a kernel density estimate to the observed rank-ordered log-transformed body masses, using the smallest bandwidth that results in a smoothed, continuous, unimodal null distribution (Silverman 1986). The kernel density estimate is transformed to a rank order versus body mass distribution by multiplying the densities by the number of species in the observed dataset. Differences in the mass between consecutive, rank ordered body masses from the observed dataset are compared to the change in rank among similar differences in body mass from the unimodal null model. This comparison generates a measure of the probability of the difference between consecutive masses in the observed dataset being significantly different from that expected from the null distribution, and thus whether the difference can be considered a discontinuity. Clusters of species between significant discontinuities are defined as aggregations. Further details of the GRI method may be found in Restrepo et al. (1997) and Wardwell et al. (2008).

For each of the datasets, a matrix of sites (columns) by $\log$ mean body mass (rows) was developed. Values of $\log$ body mass (to three decimal places) between the minimum and maximum for the community were included as separate rows. The matrix was populated using the GRI results, with 0 s for discontinuities between aggregations, and $1 \mathrm{~s}$ within aggregations (Table $3 \mathrm{~A}$ ). Patterns of discontinuities and aggregations were compared among sites using nMDS in PRIMER (Clarke 1993). ANOSIM was then used to test for statistical differences in discontinuity and aggregation patterns between sites of defined habitat types (e.g. unlogged, logged_89, logged_93 in the Borneo dataset). Euclidean distances were used to calculate the distance matrices to ensure that double zeros were included as a basis for comparing among sites, because we were interested in the discontinuity structure of the sites' respective communities (Legendre and Legendre 1998). For the Lofty Ranges bird dataset, analysis 1 was also performed using maximum body mass from the literature (Dunning Jr. 2008). This was not possible for the Borneo dataset due to lack of maximum mass data. For the two fish datasets, analysis 1 was also performed using both maximum body mass from FishBase and maximum observed body mass.
Table 3. Matrix setup for different analyses using example data. Row labels are log 10 body sizes, column titles are habitat type ( $\mathrm{Y}$ or Z) and site number (1,2 or 3). (A) analysis 1 (species-level data) and 3 (individual-level data), where data represent discontinuities (0) and aggregations (1) identified by the gap rarity index (GRI), (B) analysis 2 (species-level data) and 4 (individual-level data), where data represent $\log 10$ (abundance +1 ).

\begin{tabular}{lllllll}
\hline & $Y \_1$ & $Y \_2$ & $Y \_3$ & $Z \_1$ & $Z \_2$ & $Z \_3$ \\
\hline A) & & & & & & \\
$\mathbf{0 . 1 5 0}$ & 0 & 1 & 0 & 0 & 0 & 0 \\
$\mathbf{0 . 1 5 1}$ & 0 & 1 & 0 & 0 & 1 & 0 \\
$\mathbf{0 . 1 5 2}$ & 0 & 1 & 0 & 1 & 1 & 0 \\
$\mathbf{0 . 1 5 3}$ & 1 & 1 & 0 & 1 & 1 & 0 \\
$\mathbf{0 . 1 5 4}$ & 1 & 1 & 0 & 1 & 1 & 1 \\
$\mathbf{0 . 1 5 5}$ & 1 & 1 & 0 & 1 & 1 & 1 \\
$\mathbf{0 . 1 5 6}$ & 1 & 1 & 0 & 1 & 1 & 1 \\
$\mathbf{0 . 1 5 7}$ & 1 & 1 & 0 & 1 & 0 & 1 \\
$\mathbf{0 . 1 5 8}$ & 1 & 1 & 0 & 1 & 0 & 1 \\
$\mathbf{0 . 1 5 9}$ & 1 & 0 & 0 & 1 & 0 & 0 \\
$\mathbf{. 6}$ & $\ldots$ & $\ldots$ & $\ldots$ & $\ldots$ & $\ldots$ & $\ldots$ \\
$\mathbf{4 . 1 2 3}$ & 0 & 0 & 0 & 1 & 0 & 0 \\
$\mathbf{4 . 1 2 4}$ & 0 & 1 & 0 & 1 & 0 & 1 \\
$\mathbf{4 . 1 2 5}$ & 0 & 1 & 0 & 1 & 0 & 1 \\
$\mathbf{4 . 1 2 6}$ & 0 & 1 & 0 & 0 & 0 & 1 \\
$\mathbf{4 . 1 2 7}$ & 0 & 1 & 1 & 0 & 0 & 1 \\
$\mathbf{4 . 1 2 8}$ & 0 & 1 & 1 & 0 & 0 & 1 \\
$\mathbf{4 . 1 2 9}$ & 0 & 1 & 1 & 0 & 0 & 1 \\
$\mathbf{4 . 1 3 0}$ & 0 & 1 & 1 & 0 & 0 & 0 \\
$\mathbf{4 . 1 3 1}$ & 0 & 1 & 1 & 0 & 0 & 0 \\
$(B)$ & & & & & & \\
$\mathbf{0 . 1 5 0}$ & 0 & 0.3 & 0 & 0 & 0 & 0 \\
$\mathbf{0 . 1 5 1}$ & 0 & 0.3 & 0 & 0 & 0.3 & 0 \\
$\mathbf{0 . 1 5 2}$ & 0 & 0.3 & 0 & 1.5 & 0 & 0 \\
$\mathbf{0 . 1 5 3}$ & 0.3 & 1.5 & 0 & 0.3 & 0 & 0 \\
$\mathbf{0 . 1 5 4}$ & 0.8 & 0.8 & 0 & 0 & 0 & 1.5 \\
$\mathbf{0 . 1 5 5}$ & 0.8 & 1.4 & 0 & 1.6 & 0 & 0.3 \\
$\mathbf{0 . 1 5 6}$ & 0 & 0.3 & 0 & 0 & 1.6 & 1.8 \\
$\mathbf{0 . 1 5 7}$ & 0 & 0 & 0 & 0 & 0 & 0 \\
$\mathbf{0 . 1 5 8}$ & 0 & 0.3 & 0 & 0.3 & 0 & 0.6 \\
$\mathbf{0 . 1 5 9}$ & 0.3 & 0 & 0 & 1.5 & 0 & 0 \\
$\mathbf{. 6}$ & $\ldots$ & $\ldots$ & $\ldots$ & $\ldots$ & $\ldots$ & $\ldots$ \\
$\mathbf{4 . 1 2 3}$ & 0 & 0 & 0 & 0.3 & 0 & 0 \\
$\mathbf{4 . 1 2 4}$ & 0 & 0.8 & 0 & 0 & 0 & 0.8 \\
$\mathbf{4 . 1 2 5}$ & 0 & 0 & 0 & 1.5 & 0 & 0.9 \\
$\mathbf{4 . 1 2 6}$ & 0 & 0 & 0 & 0 & 0 & 0.3 \\
$\mathbf{4 . 1 2 7}$ & 0 & 0 & 0.3 & 0 & 0 & 0 \\
$\mathbf{4 . 1 2 8}$ & 0 & 0 & 0.4 & 0 & 0 & 0 \\
$\mathbf{4 . 1 2 9}$ & 0 & 0 & 0 & 0 & 0 & 0.3 \\
$\mathbf{4 . 1 3 0}$ & 0 & 0 & 0 & 0 & 0 & 0 \\
$\mathbf{4 . 1 3 1}$ & 0 & 0.8 & 0.5 & 0 & 0 & 0 \\
\hline & & & & & &
\end{tabular}

\section{Analysis 2 and 4}

For each dataset the abundance and modality patterns (Fig. 1B) were compared in the species size-density distribution (analysis 2) or the individual size-density relationships (analysis 4), of either the bird or fish communities, among sites of different habitat type using ANOSIM. For each dataset the abundance and modality patterns (Fig. 1B) were compared in the species size-density distribution (analysis 2) or the individual size-density relationships (analysis 4), of either the bird or fish communities, among sites of different habitat type using ANOSIM. For each dataset the matrix of sites (columns) by log body mass (rows) was populated by assigning either the species' (analysis 2) or individual' (analysis 4) $\log 10$ (abundance+1) to its respective body mass value 
(Table 3B). Patterns of abundance of different body sizes were then compared among sites using nMDS and ANOSIM. Chord distances were used to calculate the distance matrices, because this allowed comparison of the proportion of individuals recorded within body mass classes (Legendre and Legendre 1998). Pairs of sites that have peaks (and troughs) in abundance in the same size classes as well as similar proportions of individuals within size classes give the smallest chord distances, while pairs of sites that do not share overlapping modes in the abundance distribution or similar proportions of individuals in size classes give the largest distances. Analysis 2 was repeated using maximum body mass data, as detailed for analysis 1 above.

\section{Results}

\section{Analysis 1. Discontinuity patterns across species size relationships (SSRs)}

The ANOSIM results and nMDS plots show a differentiation in bird mean body size discontinuity patterns between sites in gum (black circles) and stringybark (green triangles) habitats in the Lofty Ranges dataset (Table 4A, Fig. 2A). Differences were also found between sites in logged_93 (green triangles) and unlogged (blue squares) habitats in the Borneo dataset, but not between remaining pairwise comparisons (Table 4B, Fig. 2B). The discontinuity patterns in fish mean species size relationships were not significantly different among habitats for either dataset (Table 4C-D, Fig. 2C-D). When using maximum species body mass data recorded in the literature, the Lofty Ranges bird communities showed significant differences among the two habitats (Supplementary material Appendix 1 Table a1A, Fig. A2A). The Seychelles fish communities showed no significant differences among habitats for either maximum mass recorded in FishBase, or for observed maximum mass (Supplementary material Appendix 1 Table A1C, Fig. A1B,
A2A). For the GBR dataset, there were significant differences among disturbed reefs (black circles), and both undisturbed (blue squares) and recovering (green triangles) reefs using species size relationships based on maximum mass recorded in FishBase (Supplementary material Appendix 1 Table A1D, Fig. A1C). However the $\mathrm{R}$ value for the disturbed-undisturbed reef comparison was extremely low $(\mathrm{R}=0.097)$ suggesting there is little real separation between these two groups. Differences were only found among disturbed (black circles) and undisturbed (blue squares) habitats using maximum observed species body mass distributions (Supplementary material Appendix 1 Table A1D, Fig. A2B).

\section{Analysis 2. Abundance patterns across species size-density relationships (SSDRs)}

The ANOSIM results and nMDS plots show a stronger differentiation among sites of different habitat type when the species size-density relationships of bird communities were analysed for abundance patterns (analysis 2), compared to assessment of species size relationships for discontinuity patterns (analysis 1). This outcome holds among all habitats for both the Lofty Ranges and Borneo datasets (R-values of 0.271 vs 0.115 , and 0.326 vs 0.128 , respectively; Table 4A-B, Fig. 3A-B). No significant differences were found among habitats in either fish dataset (Table 3C-D, Fig. 3C-D). When using maximum species body mass data recorded in the literature, the Lofty Ranges bird communities showed significant differences among the two habitats (Supplementary material Appendix 1 Table A1A, Fig. A3A), although this differentiation was not as strong as when mean data were used (R-values of 0.217 vs 0.271). The Seychelles fish communities showed differences among all habitats for both maximum mass recorded in FishBase and observed maximum mass (Supplementary material Appendix 1 Table A1C, Fig. A3B, A4A). In addition, global R-values were higher compared with analysis 2

Table 4. Analysis of similarities (ANOSIM) comparing the size distributions of communities for sites of different habitat type for (A) Lofty Ranges bird, (B) Borneo bird, (C) Seychelles fish and (D) Great Barrier Reef fish communities. Analysis 1: comparison of discontinuities in species mean size relationships (SSRs). Analysis 2: comparison of abundance in species mean size-density distributions (SSDRs). Analysis 3: comparison of discontinuities in individual size-density relationships (ISDRs). Analysis 4: comparison of abundance across individual size-density relationships (ISDRs). The resemblance matrices were calculated using Euclidean distances for analyses 1 and 3, and chord distances for analyses 2 and 4 .

\begin{tabular}{|c|c|c|c|c|c|c|c|c|}
\hline \multirow[b]{2}{*}{ Factor } & \multicolumn{2}{|c|}{ Analysis 1} & \multicolumn{2}{|c|}{ Analysis 2} & \multicolumn{2}{|c|}{ Analysis 3} & \multicolumn{2}{|c|}{ Analysis 4} \\
\hline & $\mathrm{R}$ & Significance & $\mathrm{R}$ & Significance & $\mathrm{R}$ & Significance & $\mathrm{R}$ & Significance \\
\hline \multicolumn{9}{|l|}{ (A) Birds - Lofty Ranges } \\
\hline Habitat & 0.115 & 0.001 & 0.271 & 0.001 & 0.205 & 0.001 & 0.212 & 0.001 \\
\hline \multicolumn{9}{|l|}{ (B) Birds - Borneo } \\
\hline Habitat & 0.128 & 0.015 & 0.326 & 0.001 & 0.114 & 0.014 & 0.268 & 0.001 \\
\hline Logged_93, Logged_89 & 0.107 & 0.084 & 0.210 & 0.006 & 0.085 & 0.129 & 0.080 & 0.106 \\
\hline Logged_93, Unlogged & 0.237 & 0.004 & 0.472 & 0.001 & 0.218 & 0.001 & 0.442 & 0.001 \\
\hline Logged_89, Unlogged & 0.005 & 0.379 & 0.274 & 0.001 & 0.015 & 0.341 & 0.224 & 0.004 \\
\hline \multicolumn{9}{|l|}{ (C) Fishes - Seychelles } \\
\hline Habitat & 0.084 & 0.223 & 0.141 & 0.128 & 0.425 & 0.005 & 0.658 & 0.001 \\
\hline Algae, Granite & & & & & 0.600 & 0.018 & 0.867 & 0.018 \\
\hline Algae, Coral & & & & & 0.427 & 0.050 & 0.825 & 0.008 \\
\hline Granite, Coral & & & & & 0.380 & 0.013 & 0.523 & 0.001 \\
\hline \multicolumn{9}{|l|}{ (D) Fishes - GBR } \\
\hline Habitat & 0.045 & 0.052 & 0.016 & 0.236 & 0.098 & 0.001 & 0.173 & 0.001 \\
\hline Undisturbed, Disturbed & & & & & 0.132 & 0.001 & 0.143 & 0.001 \\
\hline Undisturbed, Recovering & & & & & 0.111 & 0.034 & 0.294 & 0.001 \\
\hline Disturbed, Recovering & & & & & 0.025 & 0.298 & 0.112 & 0.037 \\
\hline
\end{tabular}


Birds - Lofty Ranges

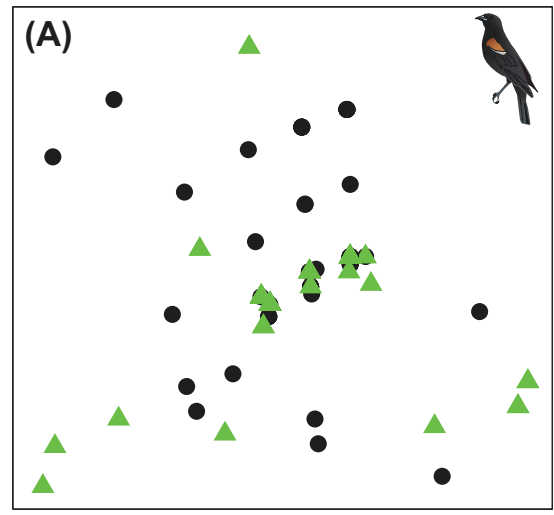

Fishes - Seychelles

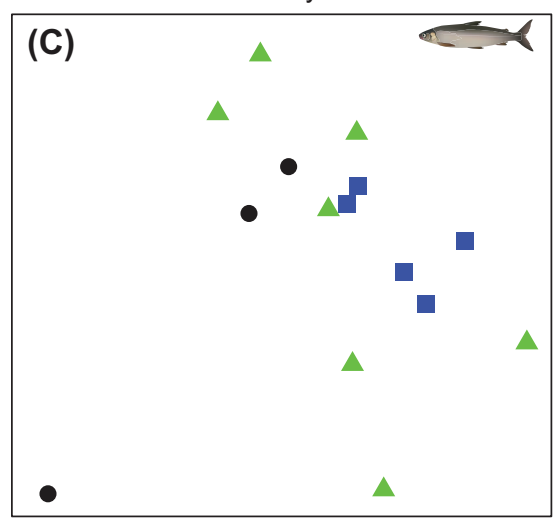

Birds - Borneo
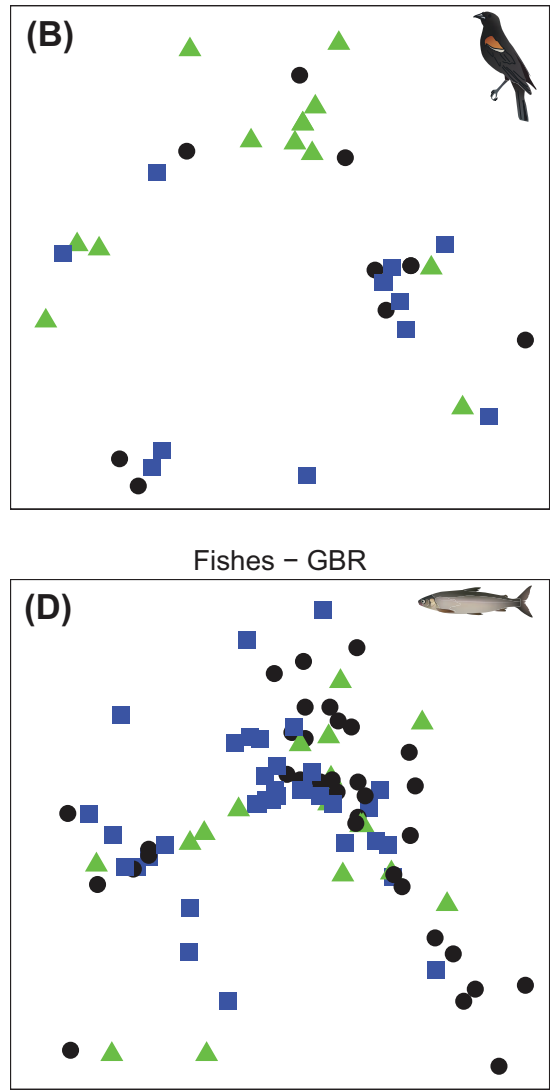

Figure 2. Non-metric multi-dimensional scaling (nMDS) comparing size distributions of communities for sites of different habitat type. Comparison of discontinuities in species size relationships (SSRs; analysis 1) for (A) Lofty Ranges bird, (B) Borneo bird, (C) Seychelles fish and (D) Great Barrier Reef fish communities. The resemblance matrices were calculated using Euclidean distances. Symbols in (A): black circles - gum woodland, green triangles - stringybark woodland; symbols in (B): black circles - logged_89 forest, green triangles - logged_93 forest, blue squares - unlogged forest; symbols in (C): black circles - algal-dominated carbonate reef, green triangles - coral-dominated carbonate reef, blue squares - granitic reef; symbols in (D): black circles - disturbed reef, green triangles - recovering reef, blue squares - undisturbed reef.

based on mean data (R-values of 0.454 and 0.337 for maximum mass in Fishbase and maximum observed mass, vs 0.141 for mean data). For the GBR dataset, differences were found among undisturbed reefs (blue squares), and both disturbed (black circles) and recovering (green triangles) reefs using either maximum mass recorded in FishBase or maximum observed species body mass (Supplementary material Appendix 1 Table A1D, Fig. A3C, A4B). However R-values were very low for the pairwise comparison between undisturbed and disturbed reefs (0.092 and 0.081 for maximum mass recorded in FishBase and observed maximum mass, respectively).

\section{Analysis 3. Discontinuity patterns across individual size-density relationships (ISDRs)}

The ANOSIM results and nMDS plots show a significant differentiation in individual bird body size discontinuity patterns between the two habitats in the Lofty Ranges dataset (Table 4A, Fig. 4A). However this differentiation was slightly weaker than for analysis 2 (R-values of 0.205 vs 0.271). Differences were also found between logged_93 (green triangles) and unlogged (blue squares) habitats in the Borneo dataset, but not between other pairwise habitat comparisons (Table 4B, Fig. 4B), and the global R-value was lower than for either analysis 1 or $2(0.114$ vs 0.128 and 0.326). There was an overall significant differentiation in individual fish body size discontinuity patterns between habitats for both datasets. For the Seychelles dataset, the ANOSIM pairwise comparisons highlight significant differences between the fish community discontinuity patterns of granite (blue squares) and both algae (black circles) and coral (green triangles) sites, but was just barely non-significant between algae (black circles) and coral (green triangles) sites (Table 4C, Fig. 4C). Furthermore, the global R-value (0.425) was higher than all previous analyses, except analysis 2 using maximum mass from the literature (0.454). Pairwise comparisons indicate significant differences in the discontinuity patterns of undisturbed (blue squares) and both disturbed (black circles) and recovering (green triangles) sites for the GBR dataset (Table 4D, Fig. 4D). The global R for the GBR dataset was greater than all previous analyses, however, it was still quite low (0.098).

\section{Analysis 4. Abundance patterns across individual size-density relationships (ISDRs)}

The ANOSIM results and nMDS plots show significant differences in the abundance patterns and modality of individual body size-density relationships between all 


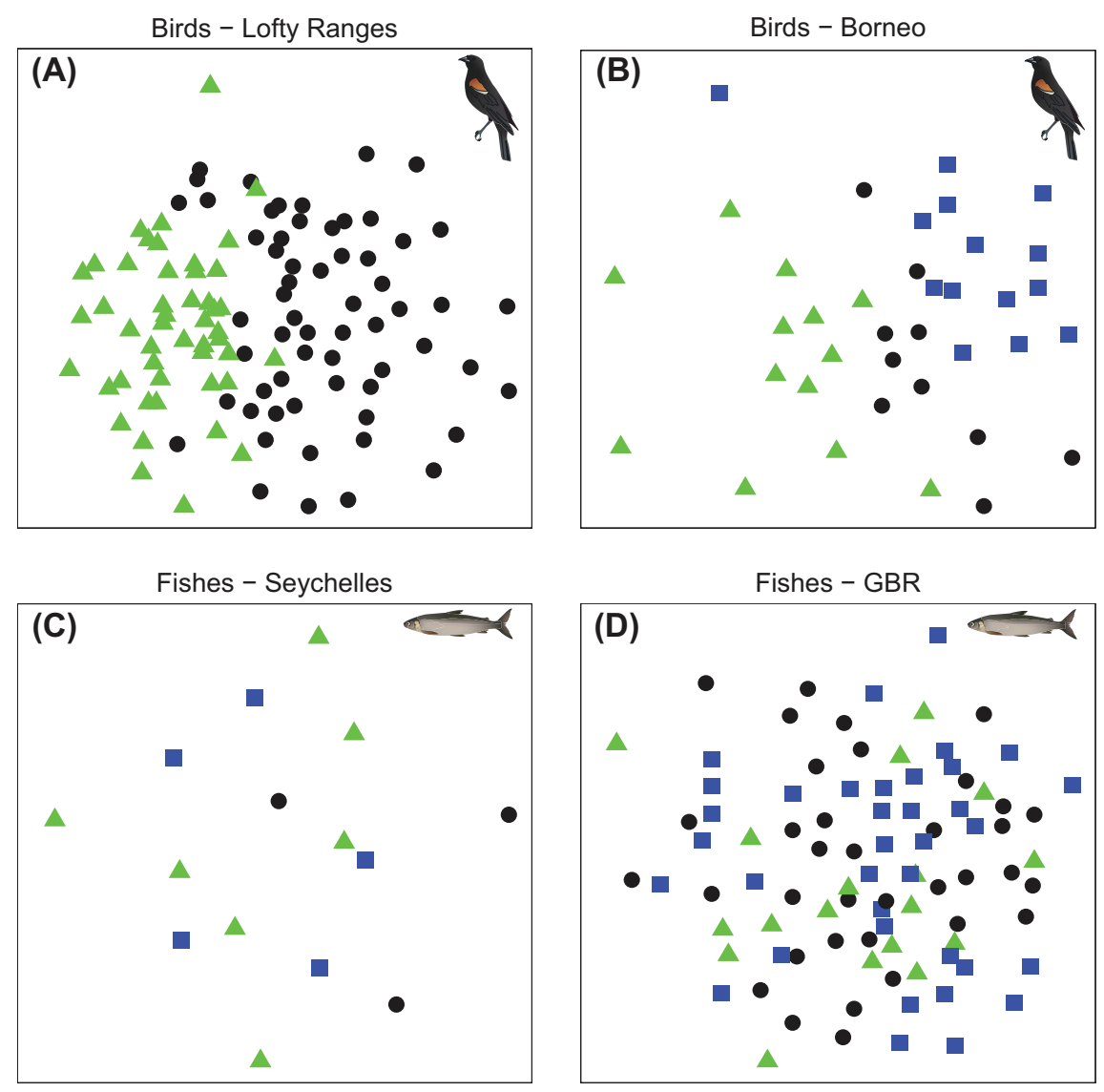

Figure 3. Non-metric multi-dimensional scaling (nMDS) comparing size distributions of communities for sites of different habitat type. Comparison of abundance patterns in species size-density relationships (SSDRs; analysis 2) for (A) Lofty Ranges bird, (B) Borneo bird, (C) Seychelles fish and (D) Great Barrier Reef fish communities. The resemblance matrices were calculated using chord distances. Symbols in (A): black circles - gum woodland, green triangles - stringybark woodland; symbols in (B): black circles - logged_89 forest, green triangles - logged_93 forest, blue squares - unlogged forest; symbols in (C): black circles - algal-dominated carbonate reef, green triangles - coral-dominated carbonate reef, blue squares - granitic reef; symbols in (D): black circles - disturbed reef, green triangles - recovering reef, blue squares - undisturbed reef.

habitats for the Lofty Ranges bird, and the Seychelles and GBR fish datasets (Table 4A, C-D, Fig. 5A, C-D). There were also significant differences between the Borneo bird communities of unlogged (blue squares) and both logged_89 (black circles) and logged_93 (green triangles) sites, but not between logged_89 and logged_93 sites (Table 5B, Fig. 5B).The differentiation among habitats for analysis 4 was greatest compared to the other three analyses for both fish datasets (R-values of 0.658 and 0.173 for the Seychelles and GBR respectively). In contrast the differentiation among habitats for analysis 4 was greater compared to analysis 3 for the bird datasets (R-values of 0.212 vs 0.205 , and 0.268 vs 0.114 for the Lofty Ranges and Borneo respectively), but were lower than for analysis 2 (R-values of 0.271 and 0.326 for the Lofty Ranges and Borneo respectively).

\section{Discussion}

This study provides a cross-ecosystem comparison of the suitability of different body size distribution indices and analyses for assessing the relationship between habitat structure and the size distributions of animal communities. Individual- or species-level size data may be used to examine this relationship in bird communities. In contrast, although there was some evidence for species-level patterns in the fish data when using maximum size metrics, the patterns were more consistent and stronger when using individuallevel data. Abundance data either at the species- (SSDRs) or individual- (ISDRs) level provides closer ties between the habitat structure and the concomitant body size distributions than when relying on species presence-absence data alone (SSRs). Significantly, individual size-density relationships (ISDRs) provide a potentially useful index for comparing drivers of body size across habitats and among taxa exhibiting determinate or indeterminate growth.

\section{Body size distributions: choice of index and analysis}

In line with previous work evaluating terrestrial body size distributions (White et al. 2007), the outcomes of this study suggest that species' mean body size provides a useful descriptive summary for bird communities. There is also potential for species' maximum body size to provide a similarly useful summary metric, although this needs further exploration with more than the single dataset (Lofty Ranges) presented in our study. There was stronger differentiation between habitat types for species size-density distributions 

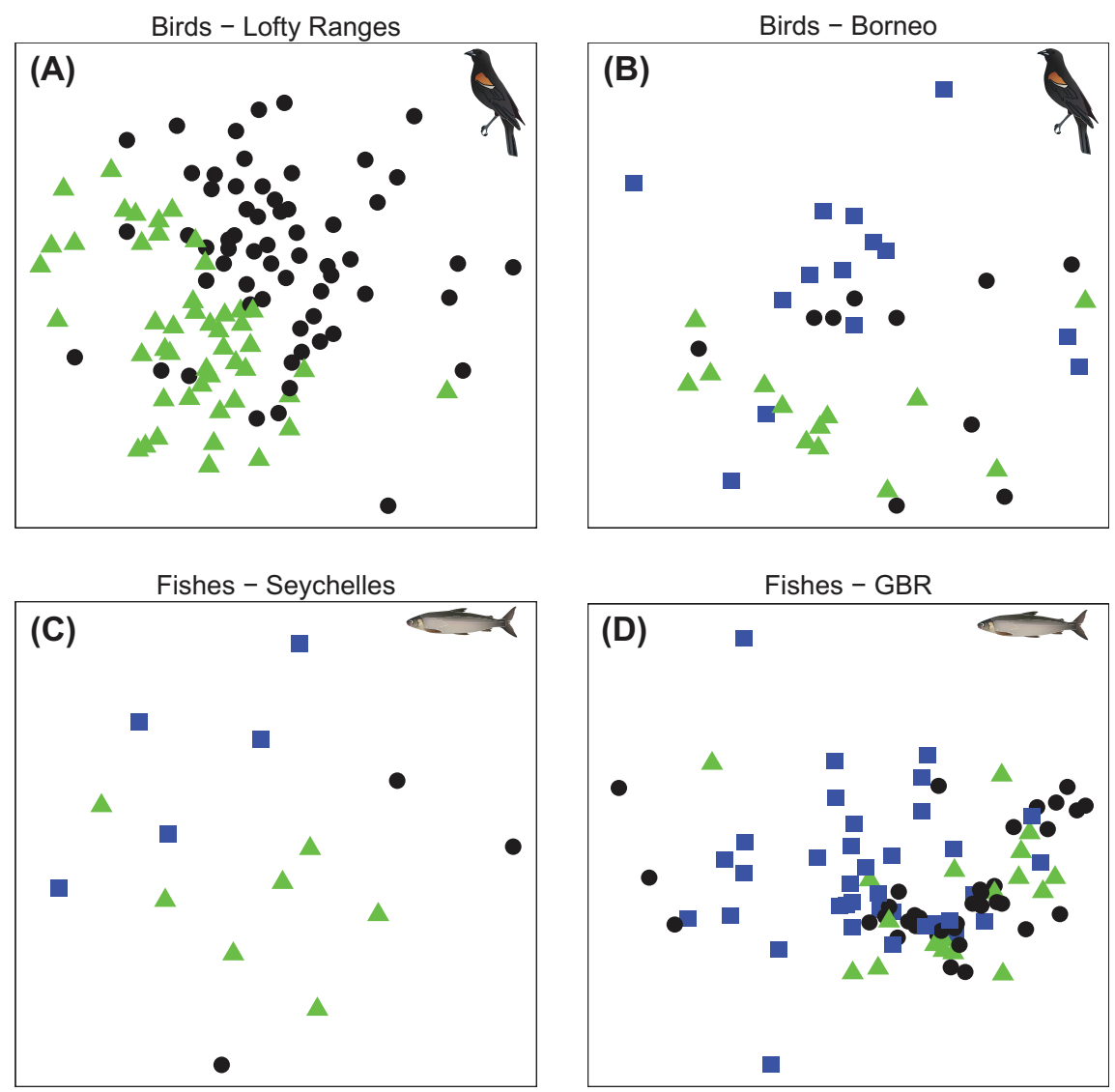

Figure 4. Non-metric multi-dimensional scaling (nMDS) comparing size distributions of communities for sites of different habitat type. Comparison of discontinuities in individual size-density relationships (ISDRs; analysis 3) for (A) Lofty Ranges bird, (B) Borneo bird, (C) Seychelles fish and (D) Great Barrier Reef fish communities. The resemblance matrices were calculated using Euclidean distances. Symbols in (A): black circles - gum woodland, green triangles - stringybark woodland; symbols in (B): black circles - logged_89 forest, green triangles - logged_93 forest, blue squares - unlogged forest; symbols in (C): black circles - algal-dominated carbonate reef, green triangles coral-dominated carbonate reef, blue squares - granitic reef; symbols in (D): black circles - disturbed reef, green triangles - recovering reef, blue squares - undisturbed reef.

(SSDRs) analysed for abundance patterns (analysis 2; Table 2) compared with species size relationships (SSRs) analysed for discontinuities (analysis 1; Table 2) in both the Borneo and Lofty Ranges dataset. This suggests that species' abundance may provide more discriminatory power with respect to habitat imposed differences in body size patterns than solely looking at species' presence-absence data (Nash et al. 2013). This finding held for species size-density relationships based on both mean and maximum mass data.

The results for the species-level fish analyses were less consistent than for birds (analyses 1 and 2). Overall, however, they suggest that mean mass does not provide a useful summary metric for fish communities when examining the relationship between habitat and body size distributions. In contrast, maximum mass observed and particularly maximum mass recorded in FishBase provide useful metrics for examining the relationship between habitat and size-density distributions (analysis 2). The inappropriateness of mean body size is not surprising considering the wide intra-specific size ranges of fish (Choat and Robertson 2002). The better performance of the maximum size metrics corresponds to existing work presenting maximum size as a good alternative to the mean as a summary metric to describe species with indeterminate growth (Jennings et al. 2001, Cumming and Havlicek 2002).

There have been recent calls to study individual sizedensity relationships (ISDRs) in terrestrial systems (Thibault et al. 2011). Mammal research often collects individual size data, however current bird (and other terrestrial animal taxa) surveys primarily collect data on species abundance, rather than information on individual size (Ernest 2013). The methods described by Thibault et al. (2011) and used in this study, extrapolating intra-specific size distributions from published mean and variance information, provide a technique for producing ISDRs without survey-derived individual size data. Our results suggest the relationships between habitat and ISDRs of birds may be detected (albeit more weakly than for the SSDRs) when assessing distribution patterns of modality within size distributions (analysis 4; Fig. 1B), despite using model-simulated individual data. However, the weaker performance of the ISDR analyses in relation to the SSDR analyses may be a function of the simulated nature of the individual-level data. Indeed, a clear caveat of this approach is that these data were extrapolated from the recorded statistics from different communities, as opposed to real data from the two locations. As a result the mean data 
Birds - Lofty Ranges

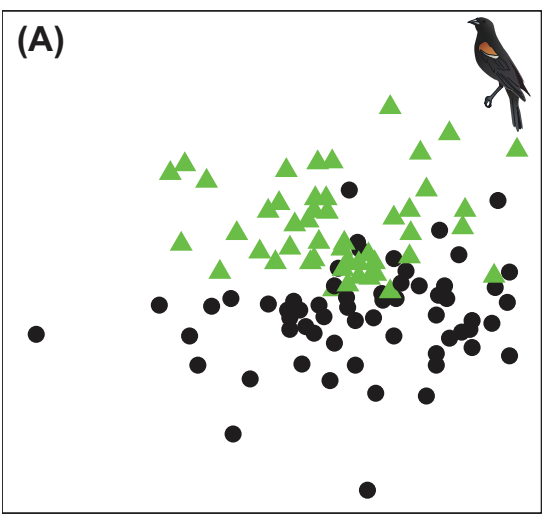

Fishes - Seychelles

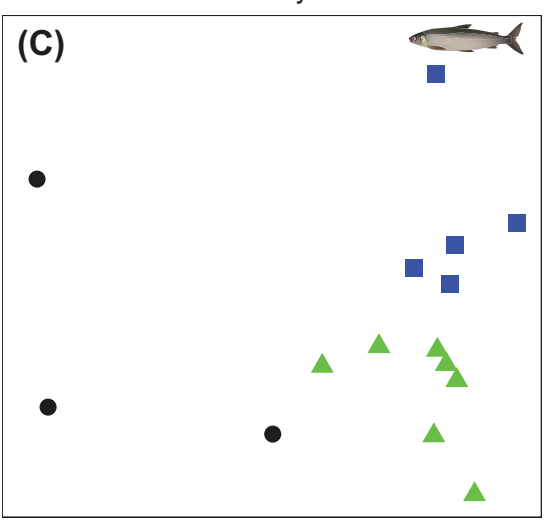

Birds - Borneo

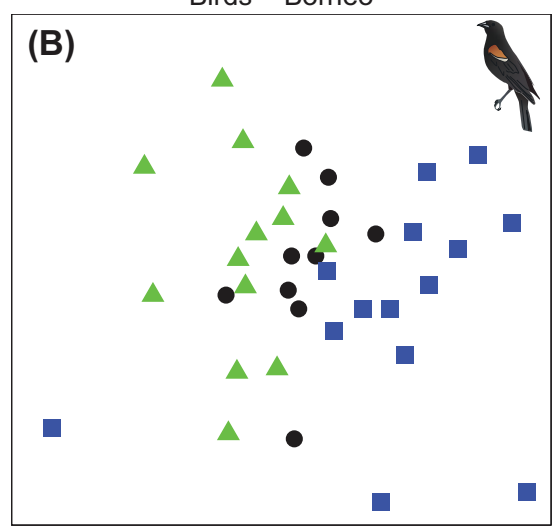

Fishes - GBR

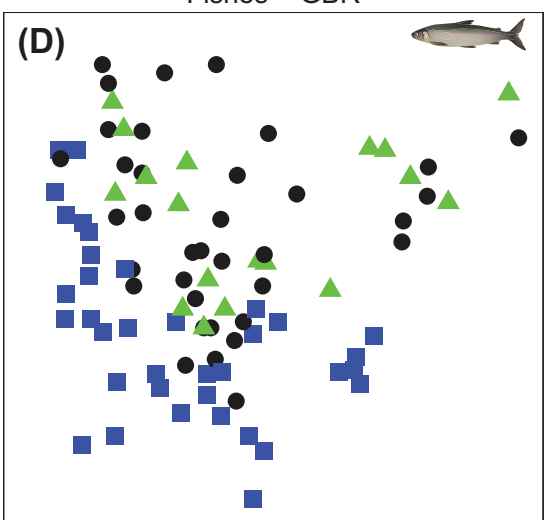

Figure 5. Non-metric multi-dimensional scaling (nMDS) comparing size distributions of communities for sites of different habitat type. Comparison of abundance patterns in individual size-density relationships (ISDRs; analysis 4) for (A) Lofty Ranges bird, (B) Borneo bird, (C) Seychelles fish and (D) Great Barrier Reef fish communities. The resemblance matrices were calculated using chord distances. Symbols in (A): black circles - gum woodland, green triangles - stringybark woodland; symbols in (B): black circles - logged_89 forest, green triangles - logged_93 forest, blue squares - unlogged forest; symbols in (C): black circles - algal-dominated carbonate reef, green triangles - coral-dominated carbonate reef, blue squares - granitic reef; symbols in (D): black circles - disturbed reef, green triangles - recovering reef, blue squares - undisturbed reef.

used in the earlier analyses may be conflated with the simulated individual data. Importantly, the assumptions used in generating the individual-level data using Thibault et al.'s (2011) approach may bias results. For example, published estimates of mean size and variance may be affected by local or latitudinal variability (Ashton 2002) resulting in deviations from the mean-variance scaling relationship employed in the method. Furthermore, individual sizes simulated in this manner may mask real discontinuities in body size distributions thus limiting the potential of discontinuity analyses on ISDRs (analysis 3), or may result in shifts in abundance along the size class axis, providing apparent differences among sites when calculating the distance matrices, which are a result of the simulation as opposed to real differences (analysis 4). Therefore our results indicate the potential for ISDRs to examine the relationship between habitat and body size distributions in bird communities, but further work is needed using survey-collected, individual size data, where available, to explore this potential further.

The relationships between habitat and fish size distributions were strongest when evaluating ISDRs (analysis 3 and 4; Table 2). This corresponds to a wide literature examining size spectra in marine communities (Jennings et al. 2001), and indicates that ISDRs are not only useful for understanding the effects of fisheries exploitation (Rice 2000), but also the potential influence of habitat change. Analyses of discontinuities within ISDRs, which ignore abundance information (analysis 3; Table 2), showed weaker relationships with habitat structure compared to analyses of abundance within ISDRs (analysis 4; Table 2), for both bird and fish communities. This corresponds to Holling's (1992) original supposition that individual-level data may mask discontinuities within body size distributions. It therefore appears that research questions and analyses aimed at examining abundance patterns and modality are more appropriate when using individual-level data (Nash et al. 2014). Nevertheless, a critical question remains regarding the mechanisms responsible for the patterns observed in size distributions. Modality and discontinuities support very different hypotheses regarding the drivers underlying the observed patterns. Multi-modality suggests a central attractor within each mode (where abundance would be greatest), whereas discontinuities suggest the existence of 'forbidden' sizes where resources are absent, and the lack of a central 
attractor within size classes such that abundance is randomly distributed within the size classes separated by discontinuities (Holling 1992, Xu et al. 2010). Rigorous tests of these two hypotheses for multiple taxa are currently lacking.

Three important considerations apply to the interpretation of our results. It is critical to tailor the choice of index and analysis to the research question, as different distributions and methods provide contrasting information regarding the distribution of resources among either individuals or species (Table 1; Robson et al. 2005). Equally, the range of drivers affecting body size distributions need to be considered. At the habitat-level scale of our analyses, competitive interactions will also influence body size distributions, and as such may mask the influence of habitat structure (Scheffer and van Nes 2006). This may partially explain why habitat effects were not seen when using certain distribution indices and analyses. Larger scale, regional datasets may provide clearer patterns across indices and analyses, and should be considered for future research. Finally, although we specifically chose taxa and systems where habitat is known to have a strong influence on body size of associated taxa, and thus the signature of habitat effects should be evident within the size distributions, it is possible that where no effect was found, that this was a function of a genuine absence of a habitat-body size relationship as opposed to a poorly performing index or analysis (suggestions of approaches to quantitatively test this further are presented in the Supplementary material Appendix 1 Text A2). We suggest that the use of multiple datasets, with consistent results within the two fish and within the two bird datasets provide support for the interpretations presented. Furthermore, these outcomes support complimentary work within the terrestrial and marine literature. As a result, we suggest that the key outcome of this study is the identification of ISDRs as a potential common currency with which to examine the relationship between habitat structure and community assembly in both terrestrial and marine systems, and among taxa exhibiting indeterminate and determinate growth. ISDRs permit cross-ecosystem comparisons, allowing clarification of the differences and similarities among marine and terrestrial systems unbiased by discipline specific approaches, and which may be more sensitive to habitat change (Ernest 2013).

\section{Size and vulnerability}

Predicting species' vulnerabilities to disturbance is of significant interest to managers as this would allow the development of appropriate mitigation strategies. Size has been presented as one trait that influences this vulnerability: large body size correlates with vulnerability to human activities such as hunting, whereas small species may be particularly susceptible to habitat loss (Owens and Bennett 2000). However, the loss of habitat structure at specific scales is likely to influence the decline of particular size classes (De la Montańa et al. 2006). Once analysis of similarities (ANOSIM) has been used to identify differences in the size distributions of communities associated with different habitat types, similarity percentages (SIMPER; Clarke 1993) may be performed on the same distance matrices used for the ANOSIM, identifying which size classes contribute to similarity among sites of a particular habitat type, and thus allow interpretation of the differences found using ANOSIM. This would allow finer scale discrimination of whether certain body sizes are likely to be susceptible to specific types of habitat change, such as losing structure at a particular scale (Nash et al. 2013). In fish communities, where individuals cover large size ranges over the course of their life (Choat and Robertson 2002), such discrimination is particularly important.

Inherent to the relationship between scale-specific disturbance of habitat and size-based vulnerability is the concept of response time, whereby different species and individuals may respond to disturbance over different time scales (Hughen et al. 2004). The body size distribution of a community is a dynamic trait, and therefore the relationship between size and vulnerability to habitat change needs ongoing evaluation (Nash et al. 2013). For example, fishes may exhibit differential loss from coral reefs in response to bleaching events: initial community changes caused by an immediate loss of live coral may be followed by distinct modifications to the community through the gradual loss of habitat structure (Graham et al. 2006). As a result, temporal studies of body size distributions are needed, in addition to the type of spatial study presented here. Once again, testing for the presence of differences among communities over time (ANOSIM) could then be followed by evaluation of which size classes are causing these changes (SIMPER).

\section{Conclusions and future directions}

There has been recent interest in comparing body size distributions across ecosystems, coincident with the desire to reconcile approaches placing greater relative weight on either size or taxonomic affinity (Petchey and Belgrano 2010). We show that size distributions of terrestrial taxa exhibiting determinate growth may be evaluated at the species or the individual-level, but incorporating abundance data across size classes adds to the robustness of these analyses. In contrast habitat driven patterns in the size distributions of aquatic taxa with indeterminate growth may be masked when using mean data. Maximum summary metrics and individual size-density relationships represent more appropriate approaches in this context. Importantly, individual size-density relationships provide a potential useful common currency with which to compare the influence of habitat structure among ecosystems. However, many questions regarding ecosystem specific differences remain unanswered. For example, there is a need to tease apart the relative influence of terrestrial versus aquatic factors compared to that of the two growth patterns. Possible examples which would allow the separation of these drivers are comparing body size distributions in insects within terrestrial soils and marine sediments (Wall et al. 2005) to understand ecosystem effects, and contrasting bird and reptile communities to assess the impact of growth pattern within a single ecosystem (Woodward et al. 2005). Other potential directions include exploring the relationships between the different body size distribution indices (Table 1), particularly for those taxa exhibiting indeterminate growth (Reuman et al. 2008). Finally, there remains considerable scope for exploration of the shape of different body size distribution indices in response to other drivers besides habitat structure, such as community 
interactions and phylogeny, which may be evident at different spatial or temporal scales (Allen et al. 2006).

Acknowledgements - This work was supported by The US Geological Survey's John Wesley Powell Centre for Analysis and Synthesis. Collection of fish data was supported by the Queensland Smart Futures Fund and the Australian Research Council. Thank you to Hugh Possingham and the Nature Conservation Society of South Australia for the use of the Lofty Ranges dataset, and to Daniel Cleary and co-authors for availability of the Borneo data. The Nebraska Cooperative Fish and Wildlife Research Unit is jointly supported by a cooperative agreement among the US Geological Survey, the Nebraska Game and Parks Commission, the University of Nebraska, the US Fish and Wildlife Service and the Wildlife Management Institute. Any use of trade names is for descriptive purposes only and does not imply endorsement by the US goverment. Fish and bird graphics courtesy of Tracey Saxby: Integration and Application Network, <http://ian.umces.edu/ imagelibrary/ $>$.

\section{References}

Allen, C. R. et al. 2006. Patterns in body mass distributions: sifting among alternative hypotheses. - Ecol. Lett. 9: 630-643.

Ashton, K. G. 2002. Patterns of within-species body size variation of birds: strong evidence for Bergmann's rule. - Global Ecol. Biogeogr. 11: 505-523.

Blackburn, T. M. and Gaston, K. J. 1994. Animal body size distributions: patterns, mechanisms and implications. - Trends Ecol. Evol. 9: 471-474.

Choat, J. H. and Robertson, D. R. 2002. Age-based studies on coral reef fishes. - In: Sale, P. F. (ed.), Coral reef fishes: dynamics and diversity in a complex ecosystem. Academic Press, Elsevier Science, pp. 57-80.

Clarke, K. R. 1993. Non-parametric multivariate analyses of changes in community structure. - Aust. J. Ecol. 18: 117-143.

Clarke, K. R. and Warwick, R. M. 2001. Change in marine communities: an approach to statistical analysis and interpretation. - PRIMER-E, Plymouth.

Cleary, D. F. R. et al. 2007. Bird species and traits associated with logged and unlogged forest in Borneo. - Ecol. Appl. 17: 1184-1197.

Cumming, G. S. and Havlicek, T. D. 2002. Evolution, ecology and multimodal distributions of body size. - Ecosyst. 5: 705-711.

De la Montańa, E. et al. 2006. Response of bird communities to silvicultural thinning of Mediterranean maquis. - J. Appl. Ecol. 43: 651-659.

DeMartini, E. E. et al. 2008. Differences in fish-assemblage structure between fished and unfished atolls in the northern Line Islands, central Pacific. - Mar. Ecol. Prog. Ser. 365: 199-215.

Doledec, S. and Statzner, B. 1994. Theoretical habitat templets, species traits and species richness: 548 plant and animal species in the Upper Rhône River and its floodplain. - Freshwater Biol. 31: 523-538.

Dunning Jr., J. B. (ed.) 2008. CRC handbook of avian body masses. - CRC Press.

Ernest, S. K. M. 2005. Body size, energy use and community structure of small mammals. - Ecology 86: 1407-1413.

Ernest, S. K. M. 2013. Using size distributions to understand the role of body size in mammalian community assembly. - In: Smith, F. A. and Lyons, S. K. (eds), Animal body size. Univ. of Chicago Press.

Fischer, J. et al. 2008. The role of landscape texture in conservation biogeography: a case study on birds in southeastern Australia. - Divers. Distrib. 14: 38-46.
Forys, E. A. and Allen, C. R. 2002. Functional group change within and across scales following invasions and extinctions in the Everglades ecosystem. - Ecosystems 5: 339-347.

Froese, R. and Pauly, D. 2012. FishBase. - <www.fishbase.org >.

Graham, N. A. J. and Nash, K. L. 2013. The importance of structural complexity in coral reef ecosystems. - Coral Reefs 32: 315-326.

Graham, N. A. J. et al. 2006. Dynamic fragility of oceanic coral reef ecosystems. - Proc. Natl Acad. Sci. USA 103: 8425-8429.

Green, A. L. and Bellwood, D. R. 2009. Monitoring functional groups of herbivorousreef fishes as indicators of coral reef resilience: a practical guide for coral reef managers in the Asia Pacific Region. IUCN working group on Climate Change and Coral Reefs. - IUCN, p. 70.

Havlicek, T. D. and Carpenter, S. R. 2001. Pelagic species size distributions in lakes: are they discontinuous? - Limnol. Oceanogr. 46: 1021-1033.

Holling, C. S. 1992. Cross-Scale morphology, geometry and dynamics of ecosystems. - Ecol. Monogr. 62: 447-502.

Hua, E. et al. 2013. Pattern of benthic biomass size spectra from shallow waters in the east China Seas. - Mar. Biol. 160: 1723-1736.

Hughen, K. A. et al. 2004. Abrupt tropical vegetation response to rapid climate changes. - Science 304: 1955-1959.

Jennings, S. et al. 2001. Weak cross-species relationships between body size and trophic level belie powerful size-based trophic structuring in fish communities. - J. Anim. Ecol. 70: 934-944.

Legendre, P. and Legendre, L. 1998. Numerical ecology. - Elsevier Science.

Nash, K. L. et al. 2013. Cross-scale habitat structure drives fish body size distributions on coral reefs. - Ecosystems 16: 478-490.

Nash, K. L. et al. 2014. Discontinuities, cross-scale patterns and the organization of ecosystems. - Ecology 95: 654-667.

O'Gorman, E. J. and Emmerson, M. C. 2011. Body mass abundance relationships are robust to cascading effects in marine food webs. - Oikos 120: 520-528.

Owens, I. P. F. and Bennett, P. M. 2000. Ecological basis of extinction risk in birds: habitat loss versus human persecution and introduced predators. - Proc. Natl Acad. Sci. USA 97: 12144-12148.

Petchey, O. L. and Belgrano, A. 2010. Body-size distributions and size-spectra: universal indicators of ecological status? - Biol. Lett. 6: 434-437.

Peters, R. H. 1983. The ecological implications of body size. - Cambridge Univ. Press.

Peterson, G. D. et al. 1998. Ecological resilience, biodiversity and scale. - Ecosystems 1: 6-18.

Possingham, M. L. et al. 2004. Species richness and abundance of birds in Mt Lofty Ranges stringybark habitat: 1999-2000 survey. - S. Aust. Ornithol. 34: 153-169.

Restrepo, C. et al. 1997. Frugivorous birds in fragmented Neotropical montane forests: landscape pattern and body mass distribution. - In: Laurance, W. F. and Bierregaard Jr., R. O. (eds), Tropical forest remnants: ecology, management and conservation of fragmented landscapes. Univ. of Chicago Press, pp. 171-189.

Reuman, D. C. et al. 2008. Three allometric relations of population density to body mass: theoretical integration and empirical tests in 149 food webs. - Ecol. Lett. 11: 1216-1228.

Rice, J. C. 2000. Evaluating fishery impacts using metrics of community structure. - ICES J. Mar. Sci. 57: 682-688.

Robson, B. J. et al. 2005. Methodological and conceptual issues in the search for a relationship between animal body-size distributions and benthic habitat architecture. - Mar. Freshwater Rev. 56: 1-11.

Scheffer, M. and van Nes, E. H. 2006. Self-organized similarity, the evolutionary emergence of groups of similar species. - Proc. Natl Acad. Sci. USA 103: 6230-6235. 
Shurin, J. B. et al. 2006. All wet or dried up? Real differences between aquatic and terrestrial food webs. - Proc. R. Soc. B 273: 1-9.

Silverman, B. W. 1986. Density estimation for statistics and data analysis. - Chapman and Hall/CRC Press.

Smith, C. and Wootton, R. 1995. The costs of parental care in teleost fishes. - Rev. Fish Biol. Fish. 5: 7-22.

Thibault, K. M. et al. 2011. Multimodality in the individual size distributions of bird communities. - Global Ecol. Biogeogr. 20: $145-153$.

Wall, D. H. et al. 2005. Soils, freshwater and marine sediments: the need for integrative landscape science. - Mar. Ecol. Prog. Ser. 304: 271-307.

Wardwell, D. A. et al. 2008. A test of the cross-scale resilience model: functional richness in Mediterranean-climate ecosystems. - Ecol. Complex. 5: 165-182.

Supplemenaty material (available online as Appendix oik-01314 at <www.oikosjournal.org/readers/appendix $>$ ). Appendix 1.
Webb, T. J. et al. 2011. The birds and the seas: body size reconciles differences in the abundance-occupancy relationship across marine and terrestrial vertebrates. - Oikos 120: 537-549.

White, E. P. et al. 2007. Relationships between body size and abundance in ecology. - Trends Ecol. Evol. 22: 323-330.

Wiens, J. A. 1989. Spatial scaling in ecology. - Funct. Ecol. 3: 385-397.

Woodward, G. et al. 2005. Body size in ecological networks. - Trends Ecol. Evol. 20: 402-409.

$\mathrm{Xu}, \mathrm{L}$. et al. 2010. Hypothesis tests on mixture model components with applications in ecology and agriculture. - J. Agric. Biol. Environ. Stat. 15: 308-326.

Yvon-Durocher, G. and Allen, A. P. 2012. Linking community size structure and ecosystem functioning using metabolic theory. - Phil. Trans. R. Soc. B 367: 2998-3007. 